\title{
Detection of millimeter-wavelength intraday variability in polarized emission from S5 0716+714
}

\author{
Jee Won Lee ${ }^{1,2}$, Sang-Sung Lee ${ }^{1,3}$, Sincheol Kang ${ }^{1,3}$, Do-Young Byun ${ }^{1,3}$, and Sungsoo S. Kim ${ }^{2}$ \\ 1 Korea Astronomy and Space Science Institute, 776 Daedeok-daero, Yuseong, Daejeon 34055, Republic of Korea \\ e-mail: sslee@kasi.re.kr \\ 2 Department of Astronomy and Space Science, Kyung Hee University, 1732, Deogyeong-daero, Giheung-gu, Yongin-si, \\ Gyeonggi-do 17104, Republic of Korea \\ 3 University of Science and Technology, 217 Gajeong-ro, Yuseong-gu, Daejeon 34113, Republic of Korea
}

Received 28 June 2016 / Accepted 25 July 2016

\begin{abstract}
We report the detection of millimeter-wavelength intraday variability in polarized emission from S5 $0716+714$ based on multifrequency polarization observations using the Korean VLBI Network (KVN) radio telescopes. The observations were conducted on November 7, 2013 at 22, 43, and $86 \mathrm{GHz}$ in dual polarization using two $21 \mathrm{~m}$ radio telescopes belonging to KVN Yonsei and Ulsan. We found significant variations in the degree of linear polarization at $86 \mathrm{GHz}$ and in polarization angle at 43 and $86 \mathrm{GHz}$ during $\sim 10 \mathrm{~h}$. We measured mean flux densities of $2.8 \mathrm{Jy}, 2.8 \mathrm{Jy}$, and $2.7 \mathrm{Jy}$ at 22,43 , and $86 \mathrm{GHz}$, respectively, with a flux modulation index ranging from $1.5 \%$ to $7.2 \%$ at the frequencies. The spectrum of the source is quite flat with spectral indices of -0.07 to $0.07 \mathrm{at} 22-43 \mathrm{GHz}$ and -0.23 to 0.04 at $43-86 \mathrm{GHz}$. The measured degree of the linear polarization ranges from $2.3 \%$ to $3.3 \%$ at $22 \mathrm{GHz}$ from $0.9 \%$ to $2.2 \%$ at $43 \mathrm{GHz}$ and from $0.4 \%$ to $4.0 \%$ at $86 \mathrm{GHz}$, yielding prominent variations at $86 \mathrm{GHz}$ over $4-5 \mathrm{~h}$. The linear polarization angle is in the range of $4^{\circ}$ to $12^{\circ}$ at $22 \mathrm{GHz},-39^{\circ}$ to $81^{\circ}$ at $43 \mathrm{GHz}$, and $66^{\circ}$ to $119^{\circ}$ at $86 \mathrm{GHz}$ with a maximum rotation of $110^{\circ}$ at $43 \mathrm{GHz}$ over $\sim 4 \mathrm{~h}$. We estimated the Faraday rotation measures (RM) ranging from -9200 to $6300 \mathrm{rad} \mathrm{m}^{-2}$ between $22 \mathrm{and} 43 \mathrm{GHz}$, and from -71000 to $7300 \mathrm{rad} \mathrm{m}^{-2}$ between 43 and $86 \mathrm{GHz}$, respectively. The frequency dependency of RM was investigated, yielding a mean power-law index, $a$, of 2.0. This implies that the polarized emission from S5 0716+714 at 22-86 GHz moves through a Faraday screen in or near the jet of the source.
\end{abstract}

Key words. galaxies: active - BL Lacertae objects: general - BL Lacertae objects: individual: S5 0716+714 - galaxies: jets radio continuum: galaxies - techniques: polarimetric

\section{Introduction}

Radio-loud active galactic nuclei (AGNs) have a relativistic jet emitting highly polarized synchrotron radiation that is ejected from a supermassive black hole (SMBH, $M_{\mathrm{BH}} \approx 10^{6}-10^{9} M_{\odot}$ ). Some of them have a fast flux variability on a timescale of hours to a day; this is called intraday variability (IDV; Heeschen et al. 1987). The IDV of an AGN was detected in the flux density at various wavelengths including in the centimeter $(\mathrm{cm})$, millimeter (mm), and optical band (Quirrenbach et al. 1991; Wagner et al. 1996; Kraus et al. 2003; Gupta et al. 2008).

The fast flux variation in the radio regime is known to be magnified by the relativistic beaming of the jet, which is moving close to the speed of light at a small angle to the line of sight (Wagner \& Witzel 1995). The IDV is commonly interpreted by intrinsic causes such as the propagation of the shock in the jet (Marscher \& Gear 1985), or by extrinsic causes such as interstellar scintillation (ISS; Rickett 1998), or both. This shows that this problem is still controversially discussed. However, intrinsic mechanisms may have an important role in the IDV flux variation at $\mathrm{mm}$ because the ISS effect becomes weaker at $\mathrm{mm}$ than at $\mathrm{cm}$. This indicates that simultaneous multiwavelength (including at the $\mathrm{cm}$ and $\mathrm{mm}$ ) observations are very important in studying the IDV of AGNs.

In addition to the flux variations, the polarization IDV was detected at $\mathrm{cm}$, yielding a larger variation amplitude in the polarized flux density than that in the total flux density (Kraus et al. 1998; Kraus et al. 2003; Fuhrmann et al. 2008). From the observations, a strong correlation was found between the polarization and the total flux density. A rapid swing of $180^{\circ}$ for the polarization angle was observed (e.g., for $0917+624$ ) when the flux density peaked, and this was interpreted as shock propagation in the jet (Quirrenbach et al. 1989). Kraus et al. (2003) suggested that the variation in polarization angle is explained by a multicomponent source or by anisotropic scattering in an inhomogeneous medium.

The BL Lac object S5 $0716+714(z=0.31 \pm 0.08$, Nilsson et al. 2008) is a bright flat spectrum source and is well known as an IDV source. The rapid flux density variation of the source was observed mainly in the radio and optical bands (Quirrenbach et al. 1991; Wagner et al. 1996; Kraus et al. 2003; Gupta et al. 2012). A correlation of the flux variability between the radio and optical bands on timescales of days was reported by Wagner et al. (1996). A transition from a one-day timescale to a slower one-week variability timescale occurred quasi-simultaneously in radio and optical. This casts doubt on ISS as the main physical cause of IDV at least in this source. A shorter timescale of about two hours for the flux variability at $6 \mathrm{~cm}$ was reported by Kraus et al. (2003). For this source, studies of the polarized emission at $\mathrm{mm}$ and its variability are still rare, however, although it is very important to investigate the sourceintrinsic mechanisms of the IDV. 
To study the intraday variability in the polarized emission of the relativistic jet of S5 $0716+714$, we performed simultaneous multifrequency polarization observations at 22,43 , and $86 \mathrm{GHz}$ during $\sim 12 \mathrm{~h}$. In this Letter, we present the results of the multifrequency simultaneous polarization observations of S5 $0716+714$ using the Korean VLBI Network (KVN), which enabled us to investigate the intraday variability of the polarization properties including the degree of polarization, electric vector position angle (EVPA), Faraday rotation measure (RM), and its frequency dependency, aiming at investigating the magnetic field environment of the inner regions of the relativistic jet.

\section{Observations and data reduction}

\subsection{Observations}

Observations were carried out at 22.4, 43.1, and $86.2 \mathrm{GHz}$ simultaneously on November 7, 2013 (MJD 56603-56 604) from UT 10:03 to 01:14 with the $21 \mathrm{~m} \mathrm{KVN}$ radio telescopes KVN Yonsei (KY) and Ulsan (KU). From the polarization observations, the four Stokes parameters, $I, Q, U$, and $V$, were measured with the KVN digital spectrometer, which has 4096 channels across a bandwidth of $512 \mathrm{MHz}$, corresponding to a channel spacing of $125 \mathrm{kHz}$ (Kang et al. 2015). Typical system temperatures, $T_{\text {sys }}$, were $85 \mathrm{~K}$ at $22 \mathrm{GHz}$ and $177 \mathrm{~K}$ at $43 \mathrm{GHz}$ at $\mathrm{KY}$ and $182 \mathrm{~K}$ at $86 \mathrm{GHz}$ at KU. A detailed description of the KVN is provided in Lee et al. (2011). The on-source integration time per measurement was 348 s (8 scans $\times 16$ iterations of on-off $\times 3 \mathrm{~s}$ ), with an on-off switching cycle like this: OFF-ON-ON-OFF-ON-OFF-OFF-ON-ON-OFF-OFFON-OFF-ON-ON-OFF-ON-OFF-OFF-ON-OFF-ON-ON-OFFOFF-ON-ON-OFF-ON-OFF-OFF-ON. To precisely measure the flux density of the source, the pointing offset correction was applied to every scan by using cross-scan observations. The time interval of the data acquisition was $40 \mathrm{~min}$, including the slewing time of the antenna. We observed Jupiter, the Crab nebula, and 3C 286 as instrumental polarization calibrator, polarization angle calibrator, and standard polarization calibrator, respectively.

\subsection{Data reduction}

We obtained single-polarization spectra $\left(v_{11^{*}}\right.$ and $\left.v_{\mathrm{rr}}\right)$ and one cross-polarization spectrum $\left(v_{\mathrm{lr}^{*}}\right.$ or $\left.v_{\mathrm{rl}^{*}}\right)$ from the single-dish polarimetric observations by using the KVN digital spectrometer backend. The polarization spectra were described by the four Stokes parameters, $I, Q, U$, and $V$ (Sault et al. 1996). By assuming that the Stokes parameters $V, Q$, and $U$ equal 0 for the nonpolarized source, the D-term was removed by observing Jupiter. Then, we estimated the four Stokes parameters of $0716+714$, and finally we obtained the total flux density, $I$, the polarization angle $\chi=(1 / 2) \times \arctan (U / Q)$ and the degree of linear polarization, $p=\sqrt{Q^{2}+U^{2}} / I$. These procedures were performed with the data processing software developed by Byun et al. (in prep.). We calibrated the observed polarization angles by observing the Crab nebula, whose polarization angle is $154 \pm 2^{\circ}$ (Flett \& Henderson 1979; Aumont et al. 2010). Detailed procedures to reduce polarization data are described in Kang et al. (2015).

We obtained the total flux density of S5 $0716+714$ by converting the opacity-corrected Stokes $I\left(T_{A}^{*}\right)$ as $S_{v}=2 k T_{A}^{*} /\left(\eta A_{g}\right)$, where $k, \eta$, and $A_{g}$ are the Boltzmann constant, aperture efficiency, and physical area of the KVN single-dish antenna, respectively. We adopted the values of $\eta$ from the KVN status

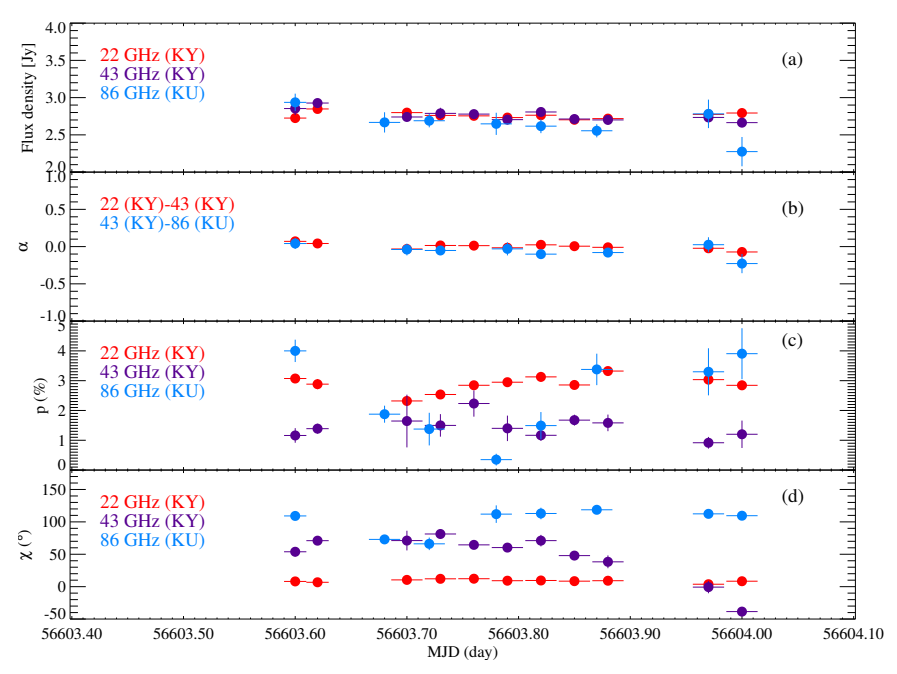

Fig. 1. Light curves of S5 $0716+714$ for a) the total flux density, b) the spectral index $\alpha$, c) the degree of linear polarization (\%), and d) the linear polarization angle. Here, the error bars indicate $1 \sigma$ of measurement error. Red, violet, and blue symbols indicate 22,43 , and $86 \mathrm{GHz}$, respectively, in panels a), c), and d). The red symbols indicate the spectral indices between 22 and $43 \mathrm{GHz}$, and the blue symbols indicate the spectral indices between 43 and $86 \mathrm{GHz}$ in panel b).

reports ${ }^{1}$. The rms errors of the total flux measurements were $37 \mathrm{mJy}, 64 \mathrm{mJy}$, and $104 \mathrm{mJy}$ at 22, 43, and $86 \mathrm{GHz}$, respectively. We obtained reliable data for $\sim 10 \mathrm{~h}$ (MJD = 56 603.60-56 604.00) due to the system setup or large pointing offsets $\left(>5^{\prime \prime}\right)$ at $86 \mathrm{GHz}$.

\section{Results}

From our multifrequency polarization observations using the KVN radio telescopes, we obtained $8-11$ measurements on the polarization of S5 $0716+714$ at 22,43 , and $86 \mathrm{GHz}$ over about $10 \mathrm{~h}$ shown in Fig. 1 and summarized in Table 1, where all errors are $1 \sigma$ errors statistically obtained from the 8-scan on-off observations. The measurements at 22 and $43 \mathrm{GHz}$ were simultaneously obtained, and those at $86 \mathrm{GHz}$ had time offsets of about half an hour with respect to those at 22 and $43 \mathrm{GHz}$ (e.g., Fig. 1a). For parameters obtained from the measurements at 22, 43 , and $86 \mathrm{GHz}$, we referred the measurement times to those of the 22 and $43 \mathrm{GHz}$ measurements (e.g., Fig. 1b). From the simultaneous multifrequency polarization observations obtained at 22, 43, and $86 \mathrm{GHz}$ on November 7, 2013 for S5 0716+714, we found that there were no significant intraday variations in flux densities. The flux densities $S_{v}$ ranged from $2.70-2.85 \mathrm{Jy}$, $2.66-2.93 \mathrm{Jy}$, and $2.27-2.94 \mathrm{Jy}$ at 22,43 , and $86 \mathrm{GHz}$, respectively. The mean flux densities $\left\langle S_{v}\right\rangle$ were $2.76 \mathrm{Jy}, 2.76 \mathrm{Jy}$, and $2.65 \mathrm{Jy}$ at 22,43 , and $86 \mathrm{GHz}$, respectively. To investigate the magnitude of the flux density variation, we estimated the modulation index $m$ defined by Kraus et al. (2003) as $m$ [\%] = $100 \sigma_{\mathrm{s}} /\langle S\rangle$, where $\sigma_{\mathrm{s}}$ represents the standard deviation of $\langle S\rangle$. The modulation indices were $1.5 \%, 2.8 \%$, and $7.2 \%$ at 22,43 , and $86 \mathrm{GHz}$, respectively, as summarized in Table 2. To evaluate the significance of a variability, we performed a $\chi_{r}^{2}$ test as in Kraus et al. (2003). For $N=11(22 / 43 \mathrm{GHz})$ and $N=8$ $(86 \mathrm{GHz})$ measurements, a source is considered to be variable if $\chi_{r}^{2}>3.0$ and $\chi_{r}^{2}>3.5$, respectively. We found that the total flux density at $43 \mathrm{GHz}$ showed a marginal variability, whereas those at 22 and $86 \mathrm{GHz}$ were non-variable. The spectral indices of the

1 http://radio.kasi.re.kr 
Table 1. Results of the multifrequency polarization observations of S5 $0716+714$. Uncertainties are $1 \sigma$.

\begin{tabular}{|c|c|c|c|c|c|c|c|c|c|c|}
\hline \multirow[b]{2}{*}{ MJD } & \multicolumn{3}{|c|}{$22 \mathrm{GHz}(\mathrm{YS})$} & \multicolumn{3}{|c|}{$43 \mathrm{GHz}(\mathrm{YS})$} & \multicolumn{4}{|c|}{$86 \mathrm{GHz}$ (US) } \\
\hline & $S_{v}(\mathrm{Jy})$ & $\chi\left(^{\circ}\right)$ & $p(\%)$ & $S_{v}(\mathrm{Jy})$ & $\chi\left({ }^{\circ}\right)$ & $p(\%)$ & MJD & $S_{v}(\mathrm{Jy})$ & $\chi\left({ }^{\circ}\right)$ & $p(\%)$ \\
\hline 56603.60 & $2.73 \pm 0.06$ & $8.1 \pm 2.6$ & $3.1 \pm 0.1$ & $2.85 \pm 0.09$ & $53.9 \pm 7.3$ & $1.2 \pm 0.3$ & 56603.60 & $2.94 \pm 0.12$ & $109.2 \pm 4.3$ & $4.0 \pm 0.4$ \\
\hline 56603.62 & $2.85 \pm 0.03$ & $6.8 \pm 1.5$ & $2.9 \pm 0.2$ & $2.93 \pm 0.03$ & $71.0 \pm 5.9$ & $1.4 \pm 0.2$ & 56603.68 & $2.67 \pm 0.14$ & $73.0 \pm 6.7$ & $1.9 \pm 0.3$ \\
\hline 56603.70 & $2.80 \pm 0.03$ & $10.4 \pm 2.8$ & $2.3 \pm 0.2$ & $2.74 \pm 0.05$ & $71.1 \pm 15.2$ & $1.6 \pm 0.9$ & 56603.72 & $2.69 \pm 0.09$ & $66.1 \pm 9.7$ & $1.4 \pm 0.6$ \\
\hline 56603.73 & $2.76 \pm 0.02$ & $12.1 \pm 1.3$ & $2.5 \pm 0.1$ & $2.79 \pm 0.07$ & $81.3 \pm 7.2$ & $1.5 \pm 0.4$ & 56603.78 & $2.65 \pm 0.15$ & $112.0 \pm 13.5$ & $0.4 \pm 0.2$ \\
\hline 56603.76 & $2.76 \pm 0.02$ & $12.3 \pm 1.9$ & $2.8 \pm 0.1$ & $2.78 \pm 0.06$ & $64.5 \pm 5.7$ & $2.2 \pm 0.4$ & 56603.82 & $2.62 \pm 0.09$ & $113.0 \pm 8.9$ & $1.5 \pm 0.5$ \\
\hline 56603.79 & $2.73 \pm 0.03$ & $9.2 \pm 3.2$ & $2.9 \pm 0.1$ & $2.70 \pm 0.06$ & $60.3 \pm 5.9$ & $1.4 \pm 0.4$ & 56603.87 & $2.56 \pm 0.09$ & $118.7 \pm 4.3$ & $3.4 \pm 0.5$ \\
\hline 56603.82 & $2.76 \pm 0.06$ & $9.5 \pm 2.5$ & $3.1 \pm 0.2$ & $2.81 \pm 0.05$ & $71.0 \pm 8.8$ & $1.2 \pm 0.2$ & 56603.97 & $2.78 \pm 0.19$ & $112.4 \pm 2.9$ & $3.3 \pm 0.8$ \\
\hline 56603.85 & $2.70 \pm 0.02$ & $8.5 \pm 1.6$ & $2.9 \pm 0.1$ & $2.71 \pm 0.05$ & $47.9 \pm 6.3$ & $1.7 \pm 0.2$ & 56604.00 & $2.27 \pm 0.20$ & $109.5 \pm 5.6$ & $3.91 \pm 0.9$ \\
\hline 56603.88 & $2.72 \pm 0.02$ & $9.14 \pm 1.5$ & $3.3 \pm 0.1$ & $2.70 \pm 0.06$ & $38.3 \pm 9.8$ & $1.6 \pm 0.3$ & & & & \\
\hline 56603.97 & $2.77 \pm 0.03$ & $3.8 \pm 3.1$ & $3.0 \pm 0.2$ & $2.73 \pm 0.03$ & $-0.7 \pm 9.5$ & $0.9 \pm 0.2$ & & & & \\
\hline 56604.00 & $2.79 \pm 0.04$ & $8.4 \pm 3.0$ & $2.8 \pm 0.2$ & $2.66 \pm 0.06$ & $-38.5 \pm 6.9$ & $1.2 \pm 0.5$ & & & & \\
\hline
\end{tabular}

Table 2. Results of the statistical analysis.

\begin{tabular}{cccccccccc}
\hline \hline $\begin{array}{c}v \\
(\mathrm{GHz})\end{array}$ & $\begin{array}{c}\left\langle S_{v}\right\rangle \\
(\mathrm{Jy})\end{array}$ & $\begin{array}{c}m_{\mathrm{s}} \\
(\%)\end{array}$ & $\chi_{r}^{2}$ & $\begin{array}{c}\langle p\rangle \\
(\%)\end{array}$ & $\begin{array}{c}m_{p} \\
(\%)\end{array}$ & $\chi_{r}^{2}$ & $\begin{array}{c}\langle\chi\rangle \\
\left({ }^{\circ}\right)\end{array}$ & $\begin{array}{c}m_{\chi} \\
\left({ }^{\circ}\right)\end{array}$ & $\chi_{r}^{2}$ \\
\hline 22.4 & 2.76 & 1.5 & 2.6 & 2.9 & 9.7 & 4.8 & 8.9 & 26.5 & 1.4 \\
43.1 & 2.76 & 2.8 & 4.1 & 1.4 & 23.6 & 1.5 & 47.3 & 76.6 & 24.4 \\
86.2 & 2.65 & 7.2 & 1.6 & 2.5 & 54.3 & 20.0 & 101.7 & 19.8 & 9.7 \\
\hline
\end{tabular}

flux densities, $\alpha\left(S_{v} \propto v^{\alpha}\right.$, where $v$ is the observing frequency), ranged from -0.072 to 0.07 between 22 and $43 \mathrm{GHz}$ and from -0.228 to -0.041 between 43 and $86 \mathrm{GHz}$. This means that the source exhibits a flat spectrum source.

The measured degree of linear polarization $p$ is in the range of $2.3 \%$ to $3.3 \%$ at $22 \mathrm{GHz}, 0.9 \%$ to $2.2 \%$ at $43 \mathrm{GHz}$, and $0.4 \%$ to $4.0 \%$ at $86 \mathrm{GHz}$. The modulation indices of the degree of linear polarization were $9.7 \%, 23.6 \%$, and $54.3 \%$ at 22,43 , and $86 \mathrm{GHz}$, respectively. We found that $p$ at $22 \mathrm{GHz}$ marginally varied with $\chi_{r}^{2}=5$ and $p$ at $86 \mathrm{GHz}$ significantly varied with $\chi_{r}^{2}=20$, decreasing by a factor of 10 from $4.0 \%$ to $0.4 \%$ in $\sim 4 \mathrm{~h}$ and then changing back to $3.9 \%$ in $\sim 5 \mathrm{~h}$. The linear polarization angle $\chi$ is in the range of $4^{\circ}$ to $12^{\circ}$ at $22 \mathrm{GHz},-39^{\circ}$ to $81^{\circ}$ at $43 \mathrm{GHz}$, and $66^{\circ}$ to $119^{\circ}$ at $86 \mathrm{GHz}$, yielding significant variations at 43 and $86 \mathrm{GHz}$ with $\chi_{r}^{2}=24$ and 10 , respectively. We found that $p$ started to rotate first in MJD 56603.60 at $86 \mathrm{GHz}$, and in MJD 56603.82 at $43 \mathrm{GHz}$. The amounts of the rotation are $43^{\circ}$ at $86 \mathrm{GHz}$, and $110^{\circ}$ at $43 \mathrm{GHz}$. At $86 \mathrm{GHz}, p$ rotates back to $\sim 110^{\circ}$ in MJD 56603.78 when $\chi$ at $86 \mathrm{GHz}$ is minimum. In particular, the rotation of $120^{\circ}$ at $43 \mathrm{GHz}$ occurred over $\sim 4 \mathrm{~h}$.

\section{Discussion and conclusions}

Multifrequency polarization observations of S5 $0716+714$ showed a polarization IDV at $\mathrm{mm}$ (not at $\mathrm{cm}$ ) with non-variable total flux density. These results may be different from the $\mathrm{cm}$ polarization variability detected at lower frequencies by Fuhrmann et al. (2008): both total and polarized flux density varied, with the polarization variability being stronger at lower frequency and faster at higher frequency. This may imply that the Faraday rotation effect plays an important role in the polarization variability at lower frequency. A cm polarization variability was also detected by Kraus et al. (2003): the polarization variability is stronger than that of total flux density by factors of 3-8 at 10-30 GHz (Kraus et al. 2003). The relatively strong and rapid variability of the linear polarization with moderate variability in the total flux density may be explained by the combination of the variability of the multiple compact jet components differently polarized within the beam size, as discussed in Bach et al. (2006). These authors found from VLA and VLBI observations

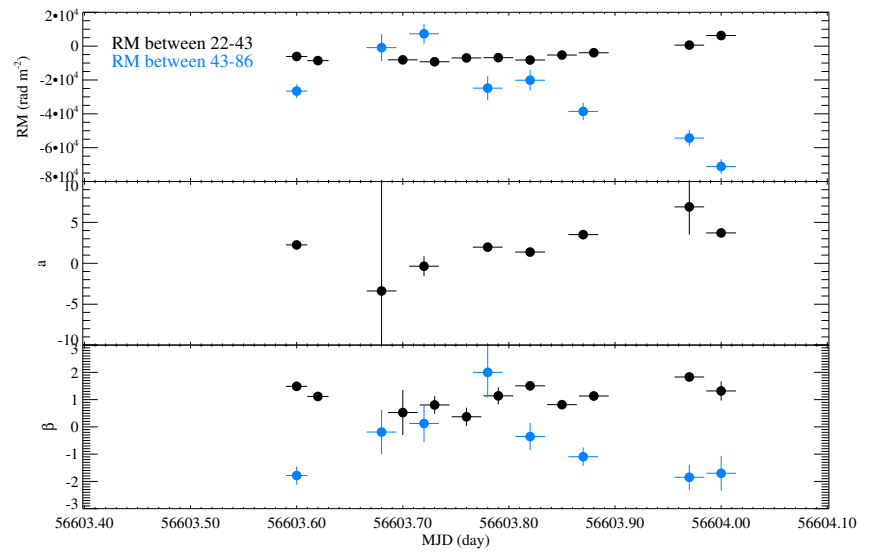

Fig. 2. Light curves of $0716+714$ for the Faraday rotation measure RM $\left(\operatorname{rad~} m^{-2}\right)$ (top), the $a$ index (middle) and the depolarization index $\beta$ (bottom). In the top panel, the black symbols indicate the rotation measure between 22 and $43 \mathrm{GHz}$ and the blue symbols indicate a rotation measure between 43 and $86 \mathrm{GHz}$. Measurements are shown only when the measurements at $86 \mathrm{GHz}$ are available to estimate the indices $a$ and $\beta$. In the bottom panel, $\beta$ between 43 and $86 \mathrm{GHz}$ is plotted at the time of $86 \mathrm{GHz}$ observed by the Ulsan telescope .

that on arcsecond scales there is no bright polarized and extended emission region. The VLA jet (on arcseconds) cannot vary so fast either because it is too extended ( $\mathrm{kpc}$ size). This means that if a polarized multicomponent structure existed, it would have to lie within the VLBI core, which implies that the multiple components may only exist on $<0.5$ milliarcsecond scales. This was nicely shown by the VSOP results, which show that the rapid polarization variability may also occur in the unresolved spaceVLBI core. One possible multicomponent models might be that two individual components that dominate the polarized emission in the beam have constant total flux and fractional polarization, and only their polarization angles change at the same velocity in either opposite or same direction. This may occur in a situation where two components move along a helical trajectory to the line of sight.

In addition to the $86 \mathrm{GHz}$ fractional polarization variability, we also found fast rotations in the linear polarization angle: $43-110^{\circ}$ at 43 and $86 \mathrm{GHz}$, with delays of $4-5 \mathrm{~h}$. The different starting time of the rotations may indicate that the polarization angle rotation at different frequencies is related with emission regions that are spatially different. Moreover, the fast rotations in linear polarization angles with a relatively moderate change in fractional polarization may be caused by rapidly variable local external Faraday rotation (e.g., Gabuzda et al. 2000). 
The polarization observations conducted simultaneously at multifrequency enabled us to accurately investigate the Faraday rotation effect on the polarized emission. Since the amount of the Faraday RM is proportional to the squares of observing wavelength $(\lambda)$ as $\chi_{\mathrm{obs}}=\chi_{0}+\mathrm{RM} \lambda_{\mathrm{obs}}^{2}$ (Jorstad et al. 2007), where $\chi_{\mathrm{obs}}$ is the observed linear polarization angle of a source and $\chi_{0}$ the intrinsic polarization angle, we may expect that the polarized emission at mm (e.g., 43 and $86 \mathrm{GHz}$ ) experience the Faraday rotation more weakly than at $\mathrm{cm}$ (e.g., $<22 \mathrm{GHz}$ ), and hence the $\mathrm{mm}$ observations are much more suitable for investigating the intrinsic linear polarization properties. From the multifrequency polarization observations of S5 $0716+714$ with $\mathrm{KVN}$, we found that the $\mathrm{RM}$ of the polarized emission from the source is in the range from -9200 to $6300 \mathrm{rad} \mathrm{m}^{-2}$ between $22 \mathrm{GHz}$ and $43 \mathrm{GHz}$ and from -71000 to $-7300 \mathrm{rad} \mathrm{m}^{-2}$ between $43 \mathrm{GHz}$ and $86 \mathrm{GHz}$, respectively, as shown in Fig. 2 (top panel).

These high $\mathrm{RM}$ values of $|\mathrm{RM}| \approx 6 \times 10^{3} \mathrm{rad} \mathrm{m}^{-2}-7 \times$ $10^{4} \mathrm{rad} \mathrm{m}^{-2}$ at the observer frame indicate that the polarized emission of S5 $0716+714$ most likely traversed extragalactic Faraday screens, since Galactic RM is as low as $20 \mathrm{rad} \mathrm{m}^{-2}$ to the direction of the source (Taylor et al. 2009). Moreover, these high RM values are factors of 10-100 higher than those observed at $15 \mathrm{GHz}$ (Hovatta et al. 2012). In addition to the rapid variation of $|R M|$, we found that the sign of RM changed. However, it is very difficult to change the sign of RM in a short time. The only way to change the sign would be to reverse the orientation of the magnetic field. There is no known physical mechanism that would explain the rapid intrinsic change of the field orientation. It is therefore very likely that the variation of integrated RM within the beam may be attributed to the rapidly variable local external Faraday rotation of multiple compact emission regions that are spatially different.

The frequency dependence of RM has been used to constrain the location of the Faraday screen in several previous studies (e.g., Kang et al. 2015; Lee et al. 2015; Algaba 2013; Jorstad et al. 2007; Trippe et al. 2012). By assuming that the Faraday rotation generates within or in close proximity of the jet, a simple jet model yielded a frequency dependence of RM as $|\operatorname{RM}(v)| \propto v^{a}$, where $v$ is the observing frequency and the power index $a$ corresponds to the power index of the evolution of electron density in jet as $n_{\mathrm{e}} \propto r^{-a}$, where $n_{\mathrm{e}}$ is the electron density and $r$ is the distance of the emitting region from the central engine. For a simple jet with spherical or conical geometries, the nominal value of the index $a=2$. This model constrained the location of a Faraday screen affecting the multifrequency polarization emission from a compact radio source: the Faraday screen may be located within or near the jet when the frequency dependence $a$ of RM is close to 2 . We therefore estimated the power-law index $a$ of the RM, yielding $a=-3.4 \sim 6.9$ with a mean of 2.0 and an rms of 2.8, as shown in Fig. 2 (middle panel). The mean value of 2.0 implies that the Faraday screen dominantly affecting the polarized emission may be located near the jet of the source. However, due to the strong variation of $a$, we cannot rule out other scalings of the electron density (Contopoulos \& Lovelace 1994).

To investigate the external Faraday depolarization effect of the polarized emission, we fit the degree of linear polarization at $22-43 \mathrm{GHz}$ and $43-86 \mathrm{GHz}$ with a power-law model $p[\%]=A \lambda^{\beta}[\mathrm{cm}]$, where $A$ is constant and $\lambda$ is a observing wavelength and, $\beta$ is polarization power-law index, suggested by Tribble (1991) and modified by Farnes et al. (2014). We obtained $\beta$ in the range of 0.4 to 1.8 between $22 \mathrm{GHz}$ and $43 \mathrm{GHz}$ and -1.8 to 2 between $43 \mathrm{GHz}$ and $86 \mathrm{GHz}$. The variation of $\beta$ at $43-86 \mathrm{GHz}$ is expected because of the large change in the degree of polarization at $86 \mathrm{GHz}$. However, the $\beta$ of $0.4-1.8$ at $22-43 \mathrm{GHz}$ implies an inverse depolarization, that is, the degree of linear polarization at lower frequency is higher than that at higher frequency. The inverse depolarization may not be easily explained by any common external depolarization models (e.g., Hovatta et al. 2012). However, as investigated by Homan (2012), internal Faraday rotation under helical or less tangled random magnetic field configurations may explain the observed inverse depolarization at $\mathrm{mm}$.

The multifrequency polarization observations of S5 0716+714 enabled us to determine that the source showed the polarization IDV at $\mathrm{mm}$, which may be explained by the rapidly variable local external Faraday rotation of multiple compact emission regions that are spatially different. To the extent that the radio emission from S5 $0716+714$ is predominantly related with a simple jet model, we may suggest that the mm-polarized emission from the source penetrated the Faraday screen located near the jet of the source, yielding high RM of $|\mathrm{RM}| \approx 6 \times 10^{3} \mathrm{rad} \mathrm{m}^{-2}-7 \times 10^{4} \mathrm{rad} \mathrm{m}^{-2}$. We expect that short $\mathrm{mm}$ VLBI observations may probe the detailed geometry of the magnetic field.

Acknowledgements. We would like to thank the anonymous referee for important comments and suggestions that have improved the manuscript. We are grateful to Thomas Krichbaum for careful reading and his kind comments on the manuscript. The KVN is a facility operated by the Korea Astronomy and Space Science Institute. The KVN operations are supported by the Korea Research Environment Open NETwork, which is managed and operated by the Korea Institute of Science and Technology Information. This work was supported by the National Research Foundation of Korea (NRF) grant funded by the Korea government (MSIP) (No. NRF-2016R1C1B2006697).

\section{References}

Algaba, J. C. 2013, MNRAS, 429, 3551

Aumont, J., Conversi, L., Thum, C., et al. 2010, A\&A, 514, A70

Bach, U., Krichbaum, T. P., Kraus, A., Witzel, A., \& Zensus, J. A. 2006, A\&A, 452,83

Contopoulos, J., \& Lovelace, R. V. E. 1994, ApJ, 429, 139

Farnes, J. S., Gaensler, B. M., \& Carretti, E. 2014, ApJS, 212, 15

Flett, A. M., \& Henderson, C. 1979, MNRAS, 189, 867

Fuhrmann, L., Krichbaum, T. P., Witzel, A., et al. 2008, A\&A, 490, 1019

Gabuzda, D. C., Kochenov, P. Y., Cawthorne, T. V., \& Kollgaard, R. I. 2000, MNRAS, 313, 627

Gupta, A. C., Cha, S.-M., Lee, S., et al. 2008, AJ, 136, 2359

Gupta, A. C., Krichbaum, T. P., Wiita, P. J., et al. 2012, MNRAS, 425, 1357

Heeschen, D. S., Krichbaum, T., Schalinski, C. J., \& Witzel, A. 1987, AJ, 94, 1493

Homan, D. C. 2012, ApJ, 747, L24

Hovatta, T., Lister, M. L., Aller, M. F., et al. 2012, AJ, 144, 105

Jorstad, S. G., Marscher, A. P., Stevens, J. A., et al. 2007, AJ, 134, 799

Kang, S., Lee, S.-S., \& Byun, D.-Y. 2015, J. Korean Astron. Soc., 48, 257

Kraus, A., Krichbaum, T. P., \& Witzel, A. 1998, in IAU Colloq. 164: Radio

Emission from Galactic and Extragalactic Compact Sources, eds. J. A.

Zensus, G. B. Taylor, \& J. M. Wrobel, ASP Conf. Ser., 144, 277

Kraus, A., Krichbaum, T. P., Wegner, R., et al. 2003, A\&A, 401, 161

Lee, S.-S., Byun, D.-Y., Oh, C. S., et al. 2011, PASP, 123, 1398

Lee, S.-S., Kang, S., Byun, D.-Y., et al. 2015, ApJ, 808, L26

Marscher, A. P., \& Gear, W. K. 1985, ApJ, 298, 114

Nilsson, K., Pursimo, T., Sillanpää, A., Takalo, L. O., \& Lindfors, E. 2008, A\&A, 487, L29

Quirrenbach, A., Witzel, A., Qian, S. J., et al. 1989, A\&A, 226, L1

Quirrenbach, A., Witzel, A., Wagner, S., et al. 1991, ApJ, 372, L71

Rickett, B. J. 1998, in IAU Colloq. 164: Radio Emission from Galactic and

Extragalactic Compact Sources, eds. J. A. Zensus, G. B. Taylor, \& J. M.

Wrobel, ASP Conf. Ser., 144, 269

Sault, R. J., Hamaker, J. P., \& Bregman, J. D. 1996, A\&AS, 117, 149

Taylor, A. R., Stil, J. M., \& Sunstrum, C. 2009, ApJ, 702, 1230

Tribble, P. C. 1991, MNRAS, 250, 726

Trippe, S., Neri, R., Krips, M., et al. 2012, A\&A, 540, A74

Wagner, S. J., \& Witzel, A. 1995, ARA\&A, 33, 163

Wagner, S. J., Witzel, A., Heidt, J., et al. 1996, AJ, 111, 2187 


\section{Appendix A: Instrumental and weather effects on the polarization observations}

To prove that the observed rapid polarization variability is physical and real, it would be necessary to show that nearby calibrator sources do not vary in a systematic manner. Unfortunately, we were not in a position to observe a secondary calibrator frequently enough, since one measurement takes about $40 \mathrm{~min}$ and hence there was a lack of the observing time. However, we observed the Crab nebula in the first and last half of the observations and found that the observed polarization angle of the Crab nebula remains constant within $\sim 1^{\circ}$ and $\sim 4^{\circ}$ at 22 and $43 \mathrm{GHz}$, respectively, at $\mathrm{KY}$ and $\sim 4^{\circ}$ at $86 \mathrm{GHz}$ at $\mathrm{KU}$. We also observed 3C 286 and found that $p=11.5 \% \pm 0.2 \%$, $12.0 \% \pm 1.5 \%$, and $11.3 \% \pm 2.0 \%$ at 22,43 , and $86 \mathrm{GHz}$, respectively, and $\chi=34.3^{\circ} \pm 0.9^{\circ}, 37.8^{\circ} \pm 1.4^{\circ}$, and $45.2^{\circ} \pm 3.8^{\circ}$ at 22,43 , and $86 \mathrm{GHz}$, respectively, which are consistent with the known $p$ and $\chi$ of $3 \mathrm{C} 286$ at each frequency.

To additionally evaluate possible instrumental effects on the polarization observations, we here discuss whether $p$ and $\chi$ are correlated with some external parameters such as parallactic angle $(\Psi)$, antenna pointing offset, and system temperatures $\left(T_{\text {sys }}\right)$. From this we can determine the effect of instruments and weather on the polarization observations. First of all, during the polarization observations, the magnitudes of the D-term measured are $2 \%(22 \mathrm{GHz}), 7 \%(43 \mathrm{GHz})$, and $1 \%(86 \mathrm{GHz})$ within a $512 \mathrm{MHz}$ bandwidth. If there is an error in the determination of the D-term, then the D-term residual may be modulated with $\Psi$. Figure A.1a shows that during the polarization observations $\Psi$ rotated from $87.4^{\circ}$ to $-99.4^{\circ}$ at $\mathrm{KY}$ and from $91.5^{\circ}$ to $-95.3^{\circ}$ at $\mathrm{KU}$. There is no systematic correlation of $\Psi$ with $p$ and $\chi$ of the two antennas, implying that any remaining instrumental error such as the D-term determination probably does not dominate the variation of $p$ and $\chi$. The D-term arises mainly from the receiving system and the antenna optics. If the D-term varies in time, then it may be caused mainly by the antenna pointing offset for the antenna optics. We corrected for the antenna pointing offest in every scan, but there may be a residual offset after the correction, which may add a D-term error to be modulated with $\Psi$. Figure A.1b presents the antenna pointing offsets in azimuth (AZ) and elevation (EL) directions at KY and KU during

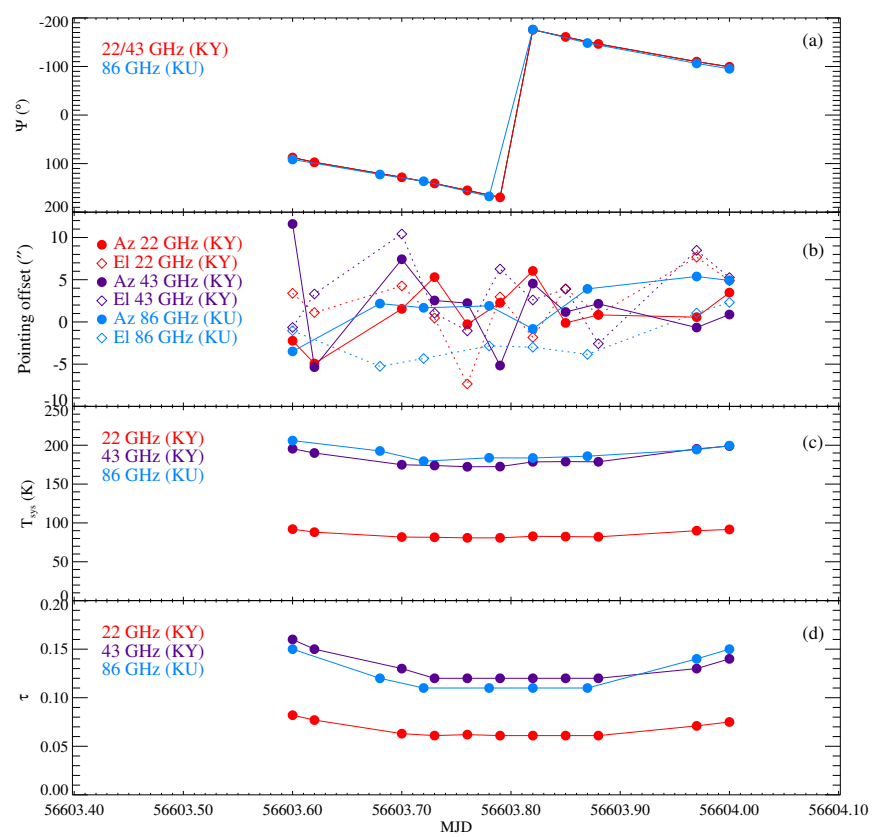

Fig. A.1. Plots of a) parallactic angle $\left({ }^{\circ}\right)$; b) antenna pointing offset $\left({ }^{\prime \prime}\right)$; c) system temperature $(\mathrm{K})$; and d) optical depth.

the observations. However, the pointing offset again shows no prominent correlation with $p$ and $\chi$ of each antenna. Furthermore, the typical error in $p$ due to the antenna pointing offset of $<5^{\prime \prime}$ may be as small as $<0.5 \%$ at $22-86 \mathrm{GHz}$ (Byun et al. in prep.). Therefore, we may rule out the instrumental effect on the strong rapid variation of $p$ and $\chi$. Although we found no significant variation in the total flux density, we may test whether atmospheric turbulence affect the data by investigating $T_{\text {sys }}$ and optical depth $(\tau)$. Figures A.1c and d show the variation in $T_{\text {sys }}$ corrected for opacity and $\tau$ in time. The atmospheric change is not strongly correlated with the total flux density. Therefore we conclude that the instrumental and weather effects on the polarization observations are very weak and do not dominate the rapid polarization variability reported here. 